\title{
A Giant Mesenteric Cyst: A Rare Entity in Childhood Surgical Spectrum
}

\author{
Sezen Ozkisacik ${ }^{1}$, Mesut Yazici $^{1}$, Nil Culhaci ${ }^{2}$, Harun Gursoy ${ }^{1}$ \\ ${ }^{1}$ Department of Paediatric Surgery, Adnan Menderes University Faculty of Medicine, Aydin, Turkey \\ ${ }^{2}$ Department of Pathology, Adnan Menderes University Faculty of Medicine, Aydin, Turkey \\ E-mail:sozkisacik@adu.edu.tr \\ Received August 9, 2010; revised August 9, 2010; accepted August 9, 2010
}

\begin{abstract}
Aim: Mesenteric cysts are rare abdominal masses which cause vomiting and distention and may be complicated with volvulus, bleeding, torsion and rupture, gastrointestinal perforation and even urinary obstruction. We present a patient with a mesenteric cyst causing gastrointestinal obstruction which treated surgically. Case: A 3 years old male patient was admitted to our hospital with symptoms of vomiting, constipation and abdominal distention. Plain abdominal anteroposterior X-ray study showed air-fluid levels and abdominal ultrasonography revealed a cystic mass of $15 \times 8 \mathrm{cms}$. Abdominal computerized tomographic examination with and without contrast material revealed a giant cystic mass with septations together with secondary findings of intestinal obstruction. Exploratory laparotomy via midline incision was made. A cystic mass containing a clear fluid was observed. The mass was obstructing the ileal passage. The cyst together with $10 \mathrm{cms}$. of ileum were removed and an ileo-ileostomy was made. Pathological examination was consistent with mesenteric cyst. He was discharged to full recovery with a symptom-free postoperative tenth day. The child is doing well at 1 year after surgery. Discussion: Mesenteric cysts, although they are rare, can be easily diagnosed modern diagnostic imaging methods and treated surgically.
\end{abstract}

Keywords: Mesenteric Cyst, Giantsurgical Teatment

\section{Introduction}

Mesenteric cyst was first observed in 1507 by an Italian anatomist, Benevienne while performing an autopsy on a 8 years-old child and first successful resection was performed by Tallaux in 1880 [1,2].

There is no pathognomonic sign and patient usually come with non-specific symptoms such as abdominal mass or acute abdominal pain $[3,4]$. Therefore these cysts are usually diagnosed during the surgery [4]. They are frequently located short segment mesentery and they may be excised without bowel resection. Surgery is usually done emergently because the symptoms are nonspecific and diagnostic evaluations and are inadequate in childhood and that's why, mortality and morbidity rates are higher than in adult [2].

We present a case with a giant mesenteric cyst with acute intestinal obstruction symptoms that had no symptoms until 3-years-old who was treated resection and ileo-ileostomy.

\section{Case Report}

A 3-years-old boy with abdominal distention, bilious vomiting and no gaita discharge reffered to our department. He had a medical therapy as constipation another hospital 3 mounths ago for a while. The patient had meconium discharge in the first 24 hours after his birth who had gaita discharge regularly once a day. His clinical findings include abdominal distention, hypoactive bowel sounds, palpable abdominal mass and normal rectal examination.

There were air-fluid levels on plain radiograph of abdomen and a giant cystic mass of $15 \times 8 \mathrm{~cm}$ was detected by emergency abdominal ultrasonography (US). Also there was a giant cystic mass with septations and intestinal obstruction findings secondary of mass by emergency abdominal computed tomography (CT). A pathological finding was not detected as a result of hemogram, blood biochemistry and urianalysis. The patient was undergone emergency surgery with prediagnosis intestinal volvulus secondary mesenteric cyst. A large cystic mass was 
found arising from the mesenteric side of the ileum which was tortioned on a clockwice during the surgery (Figure 1). After detortion of the bowel segment it was observed that the wall of the ileum which was closed configuration with the cyst was very thin (Figure 2). It was thought that this image would be due to the long time compression by the cyst on this bowel segment. The cyst with the bowel segment was resected and then primary ileo-ileostomy was done. Histologically, the cyst examination showed mesenteric cyst. The patient was discharged on the $10^{\text {th }}$ postoperative day and on his $6^{\text {th }}$ month examination any complications were detected and he is doing well at 1 year after surgery.

\section{Discussion}

The mesenteric cysts are seen at the rate of 1 in 140,000

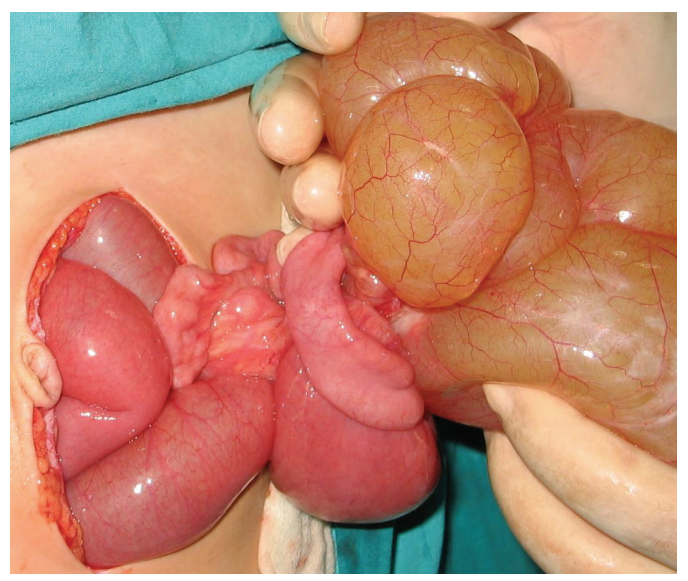

Figure 1. A large cystic mass arising from the mesenteric side of the ileum. It was tortioned on a clockwice.

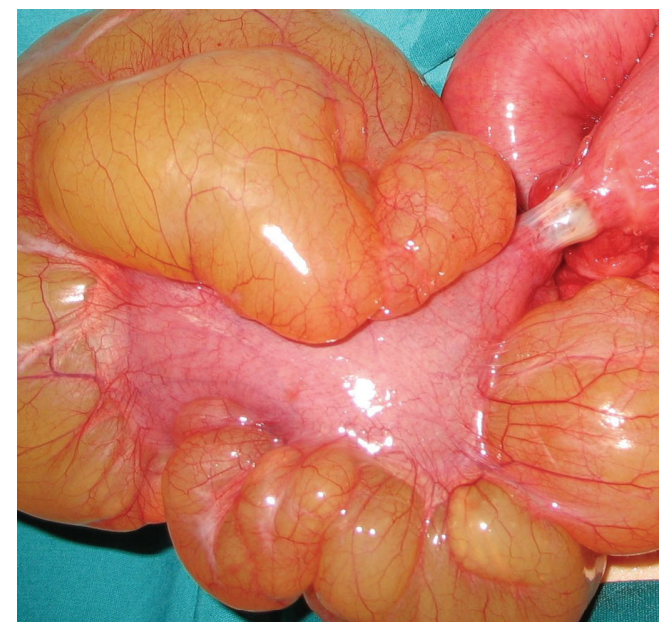

Figure 2. Operative photograph showing a giant, lobulated cyst arising from the ileal mesentery, protruding on either side. The loop of the ileum and very thin bowel wall are seen to be compressed by the cyst. in normal population and 1 in 20,000 in pediatric population and they are defined as a cystic mass located in the mesentery [5].

The symptoms are clinically pain, volvulus, vomiting, tortion, bleeding, gastrointestinal perforation, urinary obstruction and rarely cyst rupture [5]. There is no specific sign or symptom and most of the mesenteric cysts remain asymptomatic [6]. The diagnosis is usually done during the surgery incidentally [7]. Concerning this case, even a mesenteric cyst presenting with inguinal hernia was reported [1]. The diagnosis is difficult without the characteristic clinical findings and late diagnosis and inappropriate treatment sometimes occur [8].

The nature of these abdominal masses can be demonstrated with radiologic images. Preoperatively US or CT may also be useful to diagnosed these masses. With these radiologic images it is possible to show where the cyst arising from, thus help the surgeon preoperatively $[5,9]$. US is the most efficient procedure for detection of this pathology. But this method is not entirely reliable for determinate the visceral localization of cyst [10]. CT image can be better delineated adjacent organs to the cyst [6]. According to these radiologic images we thought firstly mesenteric cyst and volvulus dependent on this giant cyst of our patient. Thus, we were ready about the surgical techniques that we would do during the surgery because mesenteric cyst was in our prediagnosis. Therefore we though that the mortality and morbidity rate is lower than emergency surgery without any prediagnosis to be with radiologic images such as US or CT.

The treatment of the mesenteric cyst is excision of the cyst totally. Thus recurrence rate is very low [9]. Yet, it can not to be removed separately because of the configuration near the arteries of cyst. For this reason segmentally resection is necessary. If the segmental resection is imposible the peritoneal drainage could be done. In our case, the cyst was so large and the bowel segment was very thin near the cyst. Therefore bowel resection with giant cyst and ileo-ileostomy was performed because the single cyst resection was imposible.

Mesenteric cysts are rare lesions in childhood and they may be various size. Owing to advanced radiologic images such as US and CT these lesions can be diagnosed easily. Thus, complete surgical resection could be done with correct surgery through the advanced radiologic images even in giant mesenteric cyts.

\section{Conclusions}

In conclusion, thanks to these advanced radiological images the mesenteric cysts even the giant ones can be easily diagnosed. So the surgeons, even inexperienced surgeons handle, will ready to what will do during the sur- 
gery. By this way the mortality and morbidity rates will be lower with the usage of these diagnostic images before surgery.

\section{References}

[1] S. K. Mohanty, R. K. Bal and K. K. Maudar, "Mesenteric Cyst-An Unusual Presentation," Journal of Pediatric Surgery, Vol. 33, No. 5, 1998, pp. 792-793.

[2] S. Sander, N. Sarimurat and E. Erdoğan, "Mezenter Kistleri," Pediatrik Cerrahi Dergisi (in Turkish), Vol. 5, No. 5, 1991, pp. 144-146.

[3] R. J. Kurtz, T. M. Heimann and J. Holt, "Mesenteric and Retroperitoneal Cysts," Annals of Surgery, Vol. 203, No. 1, 1986, pp. 109-112.

[4] V. W. Vanek and A. K. Phillips, "Retroperitoneal, Mesenteric, and Omental Cysts," Archives of Surgery, Vol. 119, No. 7, 1984, pp. 838-842.
[5] W. C. Cain, S. Kennedy and N. Evans, "Renal Failure as a Result of Mesenteric Cyst," Journal of Pediatric Surgery, Vol. 39, No. 9, 2004, pp. 1440-1443.

[6] I. Mihmanli, N. Erdogan and S. Kurugoglu, "Radiological Workup in Mesenteric Cysts: Insight of a Case Report," Clinical Imaging, Vol. 25, No. 1, 2001, pp. 47-49.

[7] E. Kwan, H. Lau and W. K. Yuen, "Laparoscopic Resection of a Mesenteric Cyst," Gastrointest Endosc, Vol. 59, No. 1, 2004, pp. 154-156.

[8] N. Fujita, Y. Noda and G. Kobayashi, "Chylous Cyst of the Mesentery: US and CT Diagnosis," Abdom Imaging, Vol. 20, No. 3, 1995, pp. 259-261.

[9] M. L. Brandt, F. I. Luks and D. Filiatrault, "Surgical Indications in Antenatally Diagnosed Ovarian Cysts," Journal of Pediatric Surgery, Vol. 26, No. 3, 1991, pp. 276-281.

[10] S. Duca, M. Cazacu and L. Vlad, "Nonparasitic Abdominal Serous Cysts. A Multiple Case Report," Acta Chir Belg, Vol. 93, No. 1, 1993, pp. 18-24. 